\title{
Microstructure and Ultrastructure of Implant-Bone and Tendon-Bone Interfaces
}

\author{
Linn W. Hobbs ${ }^{1}$, Hao Wang ${ }^{1}$, Willie Mae Reese ${ }^{1}$, Bethany M. Tomerlin ${ }^{1}$, Tamara Y. C. Lim ${ }^{1}$, \\ Alexandra E. Porter ${ }^{2}$, Mark Walton ${ }^{3}$ and Nicolas J. Cotton ${ }^{4}$ \\ 1. Department of Materials Science \& Engineering, MIT, Cambridge, MA, USA \\ 2. Department of Materials, Royal School of Mines, Imperial College, London, UK \\ 3. Department of Orthopaedic Surgery, University of Otago, Dunedin, New Zealand. \\ 4. Endoscopy Divison, Smith \& Nephew, Inc., Mansfield, MA, USA
}

The musculo-skeletal system comprises a variety of both hard and soft tissue types (bone, cartilage, tendon, ligament), generative cell types (osteoblasts, chondrocytes, tenocytes, fibroblasts, all of which can derive from multipotent mesenchymal stem cell precursors), and fibrous connective-tissue proteins (chiefly collagen, types I and III) that are susceptible to varying degrees of mineralization. In the case of bone, mineralization is extensive and forms a bicontinuous composite of mineral (chiefly carbonated hydroxyapatite and precursors) and self-assembling proteins (mostly type I collagen). The important connections between tissue types are mostly hard-tissue/soft-tissue interfaces, which may involve gradients in mineralization and cell type or cell morphologies. Medical intervention in cases of orthopaedic injury - such as joint replacement, ligament replacement, or reattachment of detached tendon-invariably involves recruiting natural tissue repair processes at these interfaces, in order to affix original hard tissue (bone) to hard implants (e.g replacement femoral heads and acetabular cups for hip joint replacement, replacement condyles and tibial trays for knee joint replacement); to affix replacement soft tissue (e.g. autologous or allograft ligament or substitute autologous tendon for repair of ruptured anterior cruciate ligament in knee joints) to original hard tissue (condylar and tibial bone); or to re-affix original soft tissue (e.g. tendon) to original hard tissue (e.g. scapular bone in rotator-cuff injuries) when rupture of the original interface occurs. Electron microscopies are extending the understanding of these crucial interfaces - their microstructures and ultrastructures, their formation routes and recruitment for repair - beyond that provided by traditional light-microscope-based histology.

\section{Bone-Implant Integration}

The most studied interface re-establishment is that between bone and an orthopaedic implant made of metal (e.g. Ti-Al-V, Co-Cr alloys) or ceramic (alumina, zirconia), usually (but not always [1]) coated with an osteoconductive coating (usually hydroxyapatite (HA) [2] deposited by plasma spraying or electrodeposition [3]). The first stage in the integration of original bone and implant is essentially the same process as occurs during bone remodeling [2], which is the formation of mineralized precursor protein ribbon-like bundles (Fig. 1a) originating from osteoblasts anchored within several micrometers of the (coated) implant surface. This initial stage of developing a mineralized extracellular matrix in the gap between bone and implant occurs within three days following implantation (at least in canine and ovine in vitro models), before widespread expression of collagen, and the crystalline mineral involved does not appear to be hydroxyapatite but a lower $\mathrm{Ca} / \mathrm{P}$ ratio calcium phosphate. Only later ( 10 days) does the usual (Fig. 1b) mineralization morphology of staggered hydroxyapatite platelets decorating self-assembled collagen bundles [2] characteristic of mature bone appear. Analogous sequences appear to be followed in formation of bone-like hard tissue replacing a resorbing calcium-loaded poly(lactide co-glycolide) interference screw, used in fixation of hamstring tendon as a surrogate anterior cruciate ligament in ACL rupture repair (Fig. 1c). There is recent evidence [4] that the requisite mineralized protein is delivered prepackaged via vesicles emerging from osteoblasts early in mineralization. 


\section{Tendon-Bone Enthesis}

Re-establishment of the bone-tendon enthesis is a common procedure for reattachment of tendon, surgically re-located proximally to bone, and occurs naturally within a period of about one year. SEM and XEDS reveal quite clearly that a fibrocartilage layer, formed by chondrocyte cells on the bone surface, partially mineralizes and integrates seamlessly with bone at the fibrocartilage/bone interface (Fig. 2a). TEM reveals that the early-stage mineralization mechanism is very similar to that described above for bone mineralization (Fig. 2b). The fibrocartilage opposing tendon remains largely unmineralized, with a cell morphology gradually changing from the usual pairs of divided condrocytes to elongated cells more resembling tenocytes. The fibrocartilage layer thus forms an effective intermediate graded interface between the hard (bone) and soft (tendon) tissues.

\section{References}

[1] AE Porter, P Taak, LW Hobbs, MJ Coathup, GW Blunn, M Spector, Biomaterials 25 (2004) p. 5199. [2] V Benezra Rosen, LW Hobbs and M Spector, Biomaterials 23 (2001) p. 725; A Porter, LW Hobbs, V Benezra Rosen and M Spector, Biomaterials 23 (2001) p. 921.

[4] H Wang et al, Biomaterials 27 (2006) p. 4192; H Wang, et al, Mater. Lett. 65 (2011) 2455.

[5] S. Boonrungsiman et al, PNAS 109 (2012) p. 14170.

[6] The authors gratefully acknowledge funding from the NSF and Smith \& Nephew, Inc.
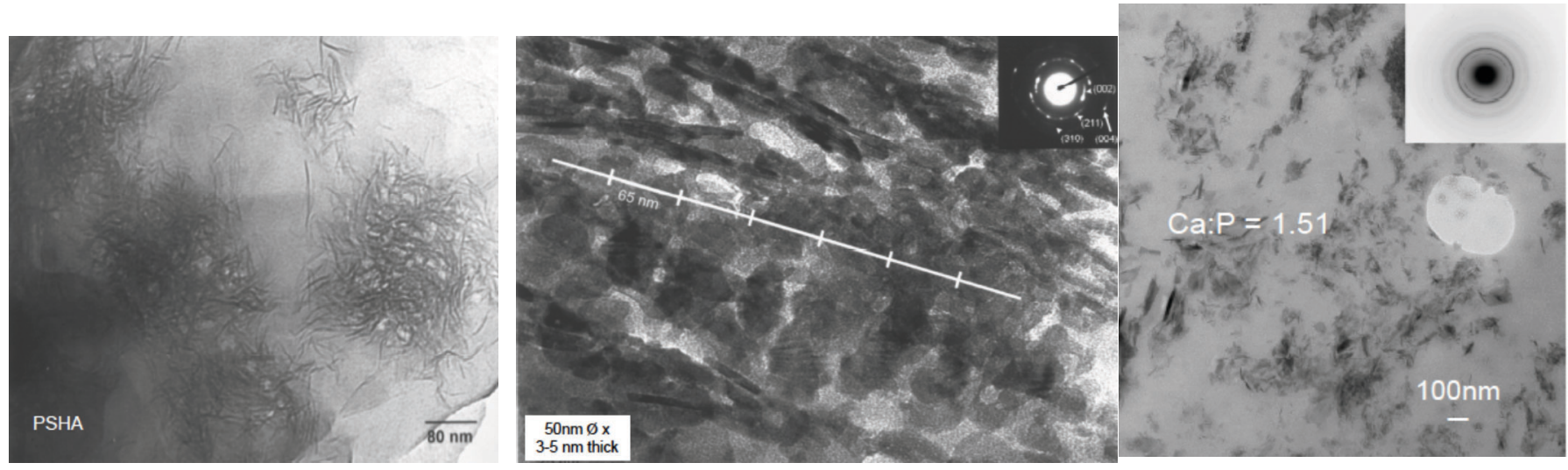

Figure 1. TEM: a) Early mineralized clusters at 3 days post implant. b) HA platelets in developing mature bone ultrastucture. c) Mineralized clusters formed after resorption of PLG interference screw.
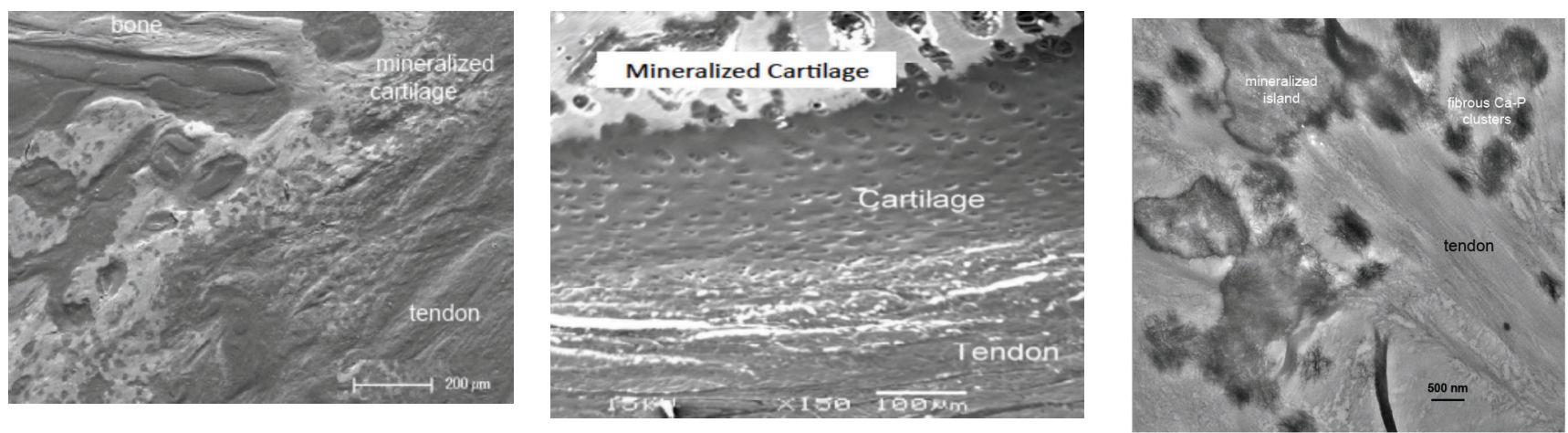

Figure 2. SEM: a) Bone/mineralized-cartilage/tendon enthesis, 12 wks. b) Natural enthesis, showing gradient in chondrocyte morphology. TEM: c) Mineralized clusters in mineralizing cartilage, 12 wks. 